\title{
Examining the Effects of Parent-Created and Parent-Implemented Video Prompting to Teach Daily Living Skills to an Adolescent with Autism
}

\author{
Gulnoza Yakubova ${ }^{1,2} \oplus \cdot$ Briella Baer Chen ${ }^{1}$
}

Accepted: 1 February 2021 / Published online: 15 February 2021

○ The Author(s), under exclusive licence to Springer Science+Business Media, LLC part of Springer Nature 2021

\begin{abstract}
Teaching parents how to create their own video-prompting (VP) and implement it to help their children learn daily living tasks at home can be empowering for parents. Using a multiple probe across three tasks design, we examined the effects of parent-created and parent-implemented VP and error correction strategy on teaching three daily living tasks to a 14-year-old child with autism spectrum disorder (ASD). Following a one-time training and continuous coaching, a parent successfully created a VP intervention for all three tasks and implemented VP with error correction with high fidelity. Following the intervention implementation, the child with ASD learned to complete daily living tasks with high levels of accuracy and maintained task completion at a 1-week follow-up.
\end{abstract}

Keywords Video prompting $\cdot$ Autism spectrum disorder $\cdot$ Parent training $\cdot$ Daily living skills

\section{Introduction}

Daily living skills are functional skills that are critical for success in adult life to function independently, and acquisition of such skills is a priority for parents of children with autism spectrum disorder (ASD; Heiman 2002). The ability to complete daily living skills independently can improve a person's quality of life, increase autonomy, and reduce dependence on family members and caregivers in daily life (Howlin and Moss 2012). Daily living skills include a range of skills necessary for independent living, such as cooking skills, self-care skills, household tasks, community skills, and many others. While there are several instructional strategies that can be effective in teaching daily living skills (Steinbrenner et al. 2020), video-based intervention (VBI) has gained increased attention due to unique features of VBI that can cater to the learning characteristics of students with ASD. VBI involves video-recorded instruction modeling the

Gulnoza Yakubova

gulnoza@umd.edu

1 University of Maryland, College Park, USA

2 Department of Counseling, Higher Education, and Special Education, University of Maryland, 3214 Benjamin Building, College Park, MD 20742, USA task completion, which a student can watch on an electronic device (e.g., computer, tablet, or a mobile phone). While there are procedural variations of VBI, such as in the type of model, or silent or audio narration of steps, VBI has been effective in teaching daily living skills to students with ASD (Aljehany and Bennett 2019; Domire and Wolfe 2015; Hong et al. 2016). There are several features of VBI that can make it effective for the acquisition of daily living skills. VBI can be used to provide individualized instruction via modeling with visual presentation and explicit instruction. Unlike in vivo modeling, VBI can provide instruction with limited distracting external stimuli by muting excess background noise and zooming in on only the relevant features of the target task (Hughes and Yakubova 2016). These features, along with the visual nature of VBI, can help accommodate for difficulties with executive functioning skills and information processing (Fleury et al. 2014).

\section{Video Prompting to Teach Daily Living Skills}

A type of VBI that can be particularly effective in completing daily living tasks by reducing cognitive overload, is video prompting (VP; Domire and Wolfe 2015). Video prompting involves a video recording of the target task, displaying the completion of the task in a step-by-step manner and may or may not involve explicit instruction with 
voice-over narration. When using VP, a student watches each VP clip, corresponding to each step of the task, and then completes that step prior to watching the next VP clip. Thus, VP can be used as a teaching intervention or self-instruction and self-prompting strategy (Shepley 2017; Shepley et al. 2018) and decrease adult prompt dependency of students with ASD (Hume et al. 2009).

The effectiveness of VP in teaching daily living skills to students with ASD is evident in numerous research studies (e.g., Aljehany and Bennett 2019; Domire and Wolfe 2015; Yakubova et al. 2019). Video prompting has been most effective for initial functional skill acquisition, particularly, the rapidity of skill acquisition and maintenance, when it was used with other instructional strategies, such as a prompting hierarchy, self-monitoring, and other strategies (Gardner and Wolfe 2015; Seaman-Tullis et al. 2019; Yakubova et al. 2019). In terms of prompting hierarchies, a system of least prompting (SLP) and most-to-least prompting strategies are commonly used to correct student errors during VP instruction. Although both prompting hierarchies can be effective in preventing future errors and promoting skill acquisition, SLP tends to be an effective strategy for faster skill acquisition compared to most-to-least prompting (Finke et al. 2017; Libby et al. 2008; Yanardag et al. 2011). While meta-analyses and systematic reviews of research examining the effects of VP in teaching functional skills to students with ASD and intellectual disability (ID) found it moderately effective (Aljehany and Bennett 2019; Park et al. 2019), VP was most effective when used with error correction procedures, particularly, for secondary schoolage children with ASD and co-occurring ID (Aljehany and Bennett 2019).

Furthermore, previous reviews and meta-analyses found that studies that used VP to teach daily living skills were primarily conducted in school settings, with VP that was created and implemented primarily by researchers (Aljehany and Bennett 2019; Domire and Wolfe 2015). Few studies examined the effects of non-researcher-created and implemented VP when teaching daily living skills in a school setting. For example, Johnson et al. (2013) trained a teacher to implement VP to teach daily living skills to students with ASD; however, the VP intervention was created by researchers. Seaman-Tullis et al. (2019) trained a teacher how to create a VP intervention and implement baseline and intervention with VP and error correction to teach a task of filling an envelope to a student with ASD. While these studies and past reviews of the VP literature found VP to be effective in improving a variety of daily living skills among students with ASD, no evidence exists on the effects of VP that is created and implemented by parents in a home setting. Examining the effects of VP to teach daily living skills with authentic intervention agents and settings (i.e., family members in a home environment where the skill is practiced and used) is critical to improve functional independence of children with ASD as they grow into adults. VP, as an evidence-based practice (EBP), can be used to provide explicit instruction in a visual format and can be created using everyday technology without requiring specialized technical training. Additionally, VP is customizable and can be created for a particular task and individual, thus, individualizing the learning experience. Yet, scarcity of research that extends VP research to examining the effects of parent-created and implemented VP in home settings is concerning.

To date, we were able to locate only one study that examined the effects of parent-delivered VP to teach daily living skills to students with ASD in a home environment (CruzTorres et al. 2020). Cruz-Torres et al. (2020) trained parents how to access and open the application that hosted the VP clip on a device, provide their child with a verbal cue to play each VP clip and complete that step of the task, and implement error correction with praise. The authors taught parents to use error correction in the form of (a) telling the child that he/she completed the step incorrectly and asking him/her to watch the video again and (b) completing that step out of the child's view if the child continued to make an error for two consecutive times within the same session. Additionally, parents were asked to provide social praise for each correctly completed step of the task to teach daily living skills to their children at home. Although this suggests that parents can successfully implement VP with error correction and social praise in home settings with their children, the research in this area remains limited. Furthermore, we were not able to identify any studies in which parents implemented VP that they had created to teach daily living skills to their child with ASD. Together, the effectiveness of parent-created and parent-implemented VP, particularly self-prompted VP, in home settings remains a gap in the existing literature.

\section{The Purpose of the Current Study}

Training parents to create and implement their own VP instruction to teach daily living skills in a home environment can be empowering for parents and provide more sustainable strategies in improving daily living independence of children with ASD as they grow into adulthood. Given the positive effects of VP on functional skills of children with ASD and scarcity of research on training parents to teach daily living skills with effective instructional strategies, it is critical to examine parent-implemented VP on the acquisition of daily living skills among children with ASD. To date, we were not able to locate a study that focused on teaching parents to create their own customized VP clips to teach a daily living skill and implement self-created VP instruction in a home setting. Therefore, the purpose of this study was to examine the effects of a parent-created and parent-implemented VP instruction with SLP on three daily living skills of an 
adolescent with ASD without a co-occurring diagnosis of ID.

\section{Methods}

\section{Participants and Setting}

The adolescent participated in the study based on the following criteria: (a) had a primary diagnosis of ASD; (b) experienced challenges with completion of daily living tasks, (c) had no vision or hearing challenges that would impede his ability to access and learn from VP instruction per parents reports; (d) had no prior experience with VP or VBI strategies; and (e) was willing to participate in the study. The parent participated in the study based on the following criteria: (a) had no prior experience with creating or implementing VP or VBI in teaching daily living or other skills to her child and (b) willingness to learn about a new EBP to teach her son daily living skills. All procedures performed in the study involving human participants were in accordance with the ethical standards of the institutional review board of the authors' institution. The parent gave informed, signed consent and the adolescent gave informed, signed assent for participation in the study prior to the start of study procedures.

Kaleb was a biracial 14-year-old male with ASD, with Caucasian and Asian ancestry. Kaleb was in 8th grade, and his primary and only spoken language was English. At the time of the study, Kaleb had a primary diagnosis of ASD according to DSM-5, and secondary diagnoses of attentiondeficit hyperactivity disorder (ADHD), language disorder, specific learning disability with impairment in written expression, and developmental coordination disorder, primarily regarding fine motor skills and handwriting. His most recent WISC-V scores indicated that he had an average IQ, scoring approximately in the 34th percentile compared to his same-age peers. The majority of his index scores fell between the 27th and 42nd percentiles, with the exception of his processing speed score, which fell at about the 70th percentile.

The parent participant, Kaleb's mother Nora, was a 51-year-old Asian-American woman, with a master's-level education. Nora was self-motivated to learn the study procedures and how to use the technology to create the VP clips. Other than performance feedback and scheduling, she needed little oversight from the research team to create the VP clips and implement study procedures. Throughout the study, she was responsive and eager to support her son's learning of new independent living skills.

All study sessions took place in the participants' home. The confirming an appointment task took place at the participants' dining room table. For each session, Kaleb was seated at the end of the table with a paper calendar, notepad, pen, cordless phone, and business card for the doctor's office (and laptop with the VP during intervention/follow-up) placed on the table in front of him. The flossing task took place in Kaleb's home bathroom, which included a sink, toilet, shower, small trashcan, and medicine cabinet-style mirror which contained the floss. During intervention and followup sessions, a small table was brought into the bathroom to hold the laptop playing the VP. The frying an egg task took place in the participants' home kitchen. Throughout the task, Kaleb used the kitchen's sink, dishwasher, fridge, trashcan, cabinets and shelves (for locating and putting away materials), and stove. The stove was a four-burner gas stove, with dials to adjust each burner's flame. During intervention and follow-up sessions, the laptop with the VP was placed on top of the microwave, on the counter, for Kaleb to watch at eye-level.

\section{Independent Variable}

The independent variable for this study was a parent-implemented intervention, which included parent-created VP and error correction in the form of SLP. The parent created one VP video for each of the three selected daily living tasks (confirming an appointment, flossing, frying an egg) based on the task analysis steps determined by the parent and research team. For each step, Nora recorded voice-over narration of herself reading the task analysis step, which was followed by the recording of her modeling the step, and then a 5-s pause (blank screen) during which her son could pause the VP to complete the step he just watched. The parent filmed the VP from third-person perspective so that her body (not just her hands) was visible in each video to model the steps. In addition, each of the VP videos were filmed in the same settings (i.e., living room table, bathroom, kitchen) and using the same materials that Kaleb would use during intervention. Each of the VP videos were filmed on a smartphone and uploaded to the mobile application (app) VivaVideo (https://www.vivavideo.tv; QuVideo Inc. 2020) to further edit and add voice-over narration. VivaVideo is a free video editing mobile application available on iOS and Android platforms. It was user-friendly for beginners to learn how to upload clips, edit, trim, and insert pauses in between each step of the task analysis to make it a VP clip. The completed VP videos were uploaded to a laptop computer for Kaleb to watch during intervention. The laptop computer was used according to Kaleb's preference; however, these VP clips could be used on many handheld electronic devices too. The duration of the VP was 2 min and $33 \mathrm{~s}$ for confirming an appointment, $3 \mathrm{~min} 44 \mathrm{~s}$ for flossing, and 5 min for frying an egg.

The intervention also included the use of error correction using SLP. If Kaleb made an error or did not initiate the step after $30 \mathrm{~s}$, Nora implemented the first level of prompting. If 
Kaleb continued to make an error or did not respond to the first level prompt after $30 \mathrm{~s}$, Nora moved to the next level of prompting. The levels of prompts included (1) instructing Kaleb to watch the VP clip again, (2) verbal prompt, (3) in-vivo model prompt, and (4) physical prompt. For verbal prompts, Nora read the task analysis step aloud. For model prompts, she performed a live model of how to complete the step. For physical prompts, she used hand-over-hand guidance to help Kaleb correctly complete the step. Nora had to use physical prompting only one time for one task throughout the intervention phase. Kaleb willingly accepted the physical prompting due to his challenge with fine motor skills and the nature of the task that involved fine motor skills. By adding SLP, Kaleb was less likely to experience frustration from repeatedly struggling with the same step over multiple sessions, primarily due to the impairment in fine motor skills. Throughout the study, Kaleb was open to his mother's use of SLP and willingly followed her guidance to improve his performance.

\section{Dependent Variable and Measurement}

The primary dependent variable was the percent of task analysis steps completed independently for each task. A step was considered independent and correct if Kaleb completed the step as shown in the VP within $30 \mathrm{~s}$ of watching the VP clip, without error correction. Two additional secondary dependent variables were also collected: percentage of steps that required error correction (via SLP) and the number of sessions required to reach mastery. Mastery criterion was set at $100 \%$ independent task completion (using VP only without error correction) for two consecutive sessions. The parent video recorded all sessions, which she then sent to the research team to collect data on the dependent variables using event recording (Ledford et al. 2018).

In selecting the tasks, Nora first consulted with her son about the three daily living tasks he was most interested in learning during that time. Nora and Kaleb generated a list of several tasks that he expressed interest in. From among the list, we eliminated the tasks that had low frequency of occurring (less than once per week) and would not fit the timing of the research design (e.g., clipping nails) or the tasks that Nora previously taught using live modeling. Then, the research team worked with Nora to select three daily living tasks of confirming an appointment, flossing, and frying an egg. These tasks were selected because they were named by the parent as tasks Kaleb could not currently complete but had expressed interest in learning how to complete. In addition, these tasks had similar levels of complexity, without the potential for crossover effects. The parent had not previously taught her son how to complete these tasks. Further, the parent was told not to ask her son to practice these tasks outside the study sessions. After the selection of tasks, the research team wrote accompanying task analyses, which Nora reviewed and further edited based on her knowledge of her child's abilities, and the materials and settings in their home. However, Kaleb was not involved in creating task analysis steps in order to prevent the potential for his learning as a result of exposure to the creation of step-by-step process of task completion before the experimental procedure. The task analysis steps for each of the tasks can be found in Table 1.

\section{Experimental Design}

A multiple probe across three behaviors/tasks design of single-case research design (SCRD) was used to identify the effects of the parent-created and implemented VP intervention with SLP on acquisition of three daily living skills of an adolescent with ASD (Gast et al. 2018). A multiple probe across tasks design is commonly used to improve desired behaviors and is more appropriate for teaching trial-based non-reversible behaviors, such as daily living skills or academic skills (Gast et al. 2018).

\section{Procedures}

Prior to beginning study sessions, Nora attended an in-person parent training session. After the training and finalization of the tasks, the parent implemented study sessions during weekends with once-a-week sessions for each of the three tasks at home with her son. Each session was video recorded by the parent and sent to the research team for data collection, fidelity checks, and feedback. The research team communicated feedback with the parent via email after each recorded session. After receiving feedback, the parent continued with the next session. In order to enhance experimental control, Nora was told not to create an opportunity for Kaleb to practice these tasks outside the study sessions.

\section{Parent Training}

Before creating the VP, the parent attended a one-time inperson training with the research team. During the training, the research team reviewed the rationale for the study and the evidence supporting VP, played a sample VP clip, and explained the study procedures, error correction, fidelity measures, and data collection schedule. Then, the research team provided a sample task analysis (cleaning a table), explained the procedure for creating a task analysis, and walked Nora through the steps of creating a VP intervention for the sample task using VivaVideo. Last, the parent and research team began to brainstorm possible tasks to be used for the study and task analysis procedures based on the child's pre-requisite skills and the nature of the tasks. The parent left the training session with printed and electronic 
Table 1 Task analyses for each of the three daily living tasks

\begin{tabular}{|c|c|c|}
\hline Confirming an appointment & Flossing & Frying an egg \\
\hline $\begin{array}{l}\text { 1. Check the calendar to find the date and time } \\
\text { of the appointment you are confirming }\end{array}$ & 1. Get the floss & $\begin{array}{l}\text { 1. Crack the egg into a bowl without breaking } \\
\text { the yolk or getting shell into the bowl }\end{array}$ \\
\hline 2. Pick up the phone & 2. Pull out a piece of floss & 2. Throw away the eggshell \\
\hline 3. Dial the number for [doctor]'s office & $\begin{array}{l}\text { 3. Pull the floss against the edge to cut and } \\
\text { remove a piece }\end{array}$ & $\begin{array}{l}\text { 3. Pour the oil into the tablespoon, and pour } \\
\text { that into the pan }\end{array}$ \\
\hline $\begin{array}{l}\text { 4. When the person or voicemail answers, say } \\
\text { "hello" }\end{array}$ & $\begin{array}{l}\text { 4. Wrap one end of the floss around your right } \\
\text { pointer finger and hold with your thumb }\end{array}$ & $\begin{array}{l}\text { 4. Turn on the stove to "medium low" and wait } \\
\text { a minute for the pan to get hot }\end{array}$ \\
\hline 5. Say "my name is [participant name]" & $\begin{array}{l}\text { 5. Hold the other end of floss with your left } \\
\text { hand, leaving about an inch of floss in } \\
\text { between }\end{array}$ & 5. Test the pan to see if it is hot \\
\hline $\begin{array}{l}\text { 6. Say "I would like to confirm my appoint- } \\
\text { ment" }\end{array}$ & $\begin{array}{l}\text { 6. One at a time, floss in between each of your } \\
\text { teeth on the upper right side of your mouth }\end{array}$ & $\begin{array}{l}\text { 6. Put the egg into the pan for several minutes } \\
\text { while it solidifies }\end{array}$ \\
\hline $\begin{array}{l}\text { 7. Say the date of the appointment, such as } \\
\text { "my appointment is on March 5th" }\end{array}$ & $\begin{array}{l}\text { 7. Wrap the used floss around your right } \\
\text { pointer finger so you have a clean section } \\
\text { of floss }\end{array}$ & $\begin{array}{l}\text { 7. Use the spatula to flip the egg so that it cooks } \\
\text { evenly }\end{array}$ \\
\hline $\begin{array}{l}\text { 8. Say the time of the appointment, such as "it } \\
\text { is at } 5 \mathrm{pm} \text { " }\end{array}$ & $\begin{array}{l}\text { 8. One at a time, floss in between each of your } \\
\text { teeth on the upper left side of your mouth }\end{array}$ & $\begin{array}{l}\text { 8. When the egg is cooked and no longer runny/ } \\
\text { translucent, turn off the stove }\end{array}$ \\
\hline 9. Say "please let me know if this is correct" & $\begin{array}{l}\text { 9. Wrap the used floss around your right } \\
\text { pointer finger so you have a clean section } \\
\text { of floss }\end{array}$ & $\begin{array}{l}\text { 9. Use the spatula to put the egg on a plate lined } \\
\text { with paper towel to soak up the excess oil }\end{array}$ \\
\hline $\begin{array}{l}\text { 10. Say "my phone number is XXX-XXX- } \\
\text { XXXX" }\end{array}$ & $\begin{array}{l}\text { 10. One at a time, floss in between each of } \\
\text { your teeth on the lower right side of your } \\
\text { mouth }\end{array}$ & $\begin{array}{l}\text { 10. Sprinkle with salt and pepper seasoning if } \\
\text { desired }\end{array}$ \\
\hline 11. Say "thank you" & $\begin{array}{l}\text { 11. Wrap the used floss around your right } \\
\text { pointer finger so you have a clean section } \\
\text { of floss }\end{array}$ & 11. Put the unused eggs back in the fridge \\
\hline 12. Say "good-bye" & $\begin{array}{l}\text { 12. One at a time, floss in between each of } \\
\text { your teeth on the lower left side of your } \\
\text { mouth }\end{array}$ & 12. Put the oil away in the cabinet \\
\hline \multirow[t]{3}{*}{ 13. Hang up the phone } & 13. Fill a cup of water, rinse out your mouth & $\begin{array}{l}\text { 13. Put the dirty dishes and utensils in the } \\
\text { dishwasher }\end{array}$ \\
\hline & 14. Throw the used floss away & $\begin{array}{l}\text { 14. Wipe out the oil from the cooled down pan } \\
\text { with paper towel and hand wash }\end{array}$ \\
\hline & 15. Put away the unused floss & 15. Use the spatula to put your egg on a plate \\
\hline
\end{tabular}

copies of all instructions and materials reviewed during the session. As described in the Dependent Variable section, the research team then worked with Nora to create task analyses for the selected tasks. Using these task analysis steps, Nora created VP videos at home with VivaVideo for each of the three tasks, which she then sent to the research team to review before starting intervention. She created each of the three VP videos independently, requiring minimal feedback from the research team beyond the initial instructions from the parent training session.

\section{Baseline}

Kaleb completed 3-5 baseline sessions for each task. At the start of each baseline session, Nora took Kaleb to the task location (e.g., kitchen, living room table, or bathroom) and began video recording the session on her smartphone. She then gave Kaleb instructions to start the task (e.g., "Show me how to floss your teeth"). To prevent frustration that Kaleb might experience due to his impairment in fine motor skills, the parent could tell Kaleb "try your best" or "it's OK if you're not sure, I just want to see what you know how to do," but otherwise did not offer any instructions, prompting, or assistance. Once Kaleb had completed the task to the best of his current ability, she offered verbal praise (e.g., "thanks for working hard!") and ended the recording. The parent then shared the recording of the session with the research team who reviewed it and offered feedback.

\section{Pre-Intervention Training}

Prior to beginning intervention sessions, Nora first trained Kaleb how to operate the VP. Using a sample VP of an unrelated task created by the research team, Nora explained how to find where the VP was located on the device, and how to select, play, pause, and rewind the VP. She then 
demonstrated the steps to the VP: play a step, pause the VP, complete the step (or rewind the VP if necessary), then play the next step of the VP. Kaleb completed the steps using the sample VP, demonstrating that he could independently selfprompt through the VP.

\section{Intervention}

Kaleb completed 6-8 intervention sessions per task. At the start of each intervention session, Nora took Kaleb to the task location and began video recording the session. She then gave Kaleb a laptop computer with the VP and instructed him to use the VP to complete the task (e.g., "I want you to show me how to fry an egg using the VP. Watch it, and follow along with the steps, rewinding if you need to"). Throughout the intervention, if Kaleb failed to initiate a step within $30 \mathrm{~s}$ of watching the VP clip or completed a step incorrectly, Nora implemented SLP, first by asking him to watch the VP clip again, and then using verbal, model, or physical prompts, increasing the level of support as needed. Once Kaleb completed the task, Nora gave verbal praise (e.g., "great job!") and ended the recording. The parent then shared the recording of the session with the research team who reviewed it and offered feedback.

\section{Follow-Up}

Kaleb completed 3 follow-up sessions for each task, after a 1 -week break from completing the task. For follow-up sessions, Nora brought Kaleb to the task location, began video recording the session, and gave Kaleb instructions to start the task. Kaleb was positive, self-motivated, and eager to self-fade the VP once he had learned the steps. Kaleb had access to the VP and was given the option to use it if he wanted to. Kaleb was told that he could watch the VP clip at the beginning of each follow-up session prior to working on the task or at any time during the task completion if he needed a reminder on how to complete the following step of task. However, error correction (SLP) or any other prompting or guidance was not used during follow-up sessions. Once Kaleb completed the task, the parent gave verbal praise and ended the recording. The parent then shared the recording of the session with the research team who reviewed it and offered feedback.

\section{Interobserver Agreement and Procedural Reliability}

A second trained observer reviewed $100 \%$ of the video recordings per phase for each task to evaluate the percent of task analysis steps completed independently. The interobserver agreement (IOA) was calculated by dividing the number of agreements by the number of agreements plus disagreements and then multiplying by 100 . This resulted in $100 \%$ IOA per task per each phase.

During the parent training session, the research team reviewed a procedural reliability checklist with the parent and provided copies to take home. The procedural reliability checklist included the following steps for Nora to complete during study sessions: (1) ensure all materials are available, (2) provide direction to complete the task, (3) give your child the device with the VP (for intervention), (4) tell your child to watch the VP (for intervention), (5) ensure that your child watches the VP (for intervention), (6) do not provide assistance or error correction (for baseline or follow-up sessions), (7) if your child looks at you or asks for confirmation, only provide neutral responses, such as "try your best," (8) if your child requires assistance during intervention, tell him to watch the VP clip again, (9) if your child requires further assistance during intervention, provide SLP, and (10) provide your child with verbal praise at the end of each task. Using the checklist, each of the authors reviewed the session video recordings and evaluated the parent's procedural reliability for $100 \%$ of sessions. Procedural reliability was calculated by dividing the number of steps completed correctly by the total number of steps and multiplying by $100 \%$. The research team discussed and resolved the disagreements from each of their evaluations of procedural reliability and reached the agreement on the percentage of procedural reliability steps followed. Procedural reliability results are displayed in Table 2. The most common errors in the procedural reliability were remembering to offer verbal praise at the end of each session and telling the child to re-watch the VP clip before beginning the error correction procedures during intervention sessions (e.g., correcting the child with a verbal prompt instead of first telling him to re-watch the VP clip).
Table 2 Mean procedural reliability score and range for the parent's implementation of each task over baseline, intervention, and follow-up phases

\begin{tabular}{lccc}
\hline & $\begin{array}{l}\text { Confirming an appointment } \\
\text { Mean (range) }\end{array}$ & $\begin{array}{l}\text { Flossing } \\
\text { Mean (range) }\end{array}$ & $\begin{array}{l}\text { Frying an egg } \\
\text { Mean (range) }\end{array}$ \\
\hline Baseline & $M=100 \%(100 \%)$ & $M=88 \%(75-100 \%)$ & $M=95 \%(75-100 \%)$ \\
Intervention & $M=93 \%(67-100 \%)$ & $M=100 \%(100 \%)$ & $M=93 \%(89-100 \%)$ \\
Follow-up & $M=92 \%(75-100 \%)$ & $M=100 \%(100 \%)$ & $M=92 \%(75-100 \%)$ \\
\hline
\end{tabular}




\section{Social Validity}

After all sessions were complete, the research team met with Nora and Kaleb to ask them questions about the study. Kaleb was asked about what he liked or did not like about the study, whether it was easy to use VP, and if he would like to continue using VP to learn other tasks in the future. Nora was asked about what she liked and did not like about the intervention, how easy or difficult it was to create and implement the intervention, whether there were aspects of the study she would change, and whether she would like to continue using VP to teach her child daily living tasks. The questions were open-ended and asked and answered verbally.

\section{Data Analyses}

We used visual analysis as the primary method of data analyses and supplemented the analyses with the effect size calculations. Visual analysis is the foundational standard of data analyses in SCRD and is used to determine the presence or absence of a functional relation between the intervention and outcome variable (Barton et al. 2018). We systematically analyzed data within and across adjacent phase and examined level, trend, and stability per process described in Barton et al. (2018). To analyze the stability of data, we used the $80-25$ rule, which meant data were considered stable if $80 \%$ of the data appeared within $25 \%$ of the median in each phase. We calculated trend using the split-middle technique and described as accelerating, decelerating, or zero-celerating. To supplement visual analysis, we calculated the effect size using Tau-U method (Parker et al. 2011). Tau-U is widely used as a non-parametric measure of effect size in SCRD studies, although it has inherent limitations and does not capture the individual variability within a phase and immediacy of effect. We interpreted Tau-U results using Parker and Vannest (2009) guidelines: The range score of Tau-U is 0 to 1.0 and can be defined as weak effect $(0-0.65)$, medium to high effect $(0.66-0.92)$ and strong effect $(0.93-1.0)$. To calculate the Tau-U effect size, we used an online effect size calculator for SCRD data (http://www.singlecaseresearch. org/calculators/tau-u; Vannest et al. 2016).

\section{Results}

Figure 1 demonstrates the percentage of steps that Kaleb completed independently (without SLP) for each of the three tasks. The mean percentage of steps that required SLP during intervention per task, and the number of sessions required to reach mastery criterion are displayed in Table 3 . Table 4 presents the steps correctly completed by the parent for creating VP clips. Table 5 includes Tau-U scores for all skills. Effect size calculation resulted in a Tau-U score of 1 between baseline-intervention and baseline-follow-up phases for each of the three skills. Readers should use caution when interpreting Tau- $\mathrm{U}$ results, as Tau- $\mathrm{U}$ is supplementary to visual analysis. While Tau-U results show overall strong effects of the intervention, they do not communicate the variability in the completion of each task.

For the confirming an appointment task, Kaleb had a mean baseline performance of $30.77 \%$ with a low, completely stable trend across the three baseline sessions. Once intervention was introduced, Kaleb demonstrated an immediate increase in percentage of steps completed independently, with a relatively stable trend across the six intervention sessions. Kaleb demonstrated a mean of $92.31 \%$ independent task completion during intervention, ranging $85-100 \%$, with a mean change of $61.54 \%$ from baseline and no overlapping data points. During follow-up, Kaleb's mean independent task completion was $92.31 \%$, ranging $85-100 \%$, with a relatively stable trend. For follow-up, Kaleb performed the task with the VP only (no SLP), with the option to use the VP as needed. Across the three follow-up sessions, Kaleb chose to use the VP only for certain steps, correctly performing an average of 5.7 out of 13 steps without using the VP (range $=1-10)$.

For the flossing task, Kaleb completed four baseline sessions with a mean of $15.0 \%$ of steps completed independently and a low, stable trend ranging 13-20\%. During intervention, Kaleb's mean independent task completion was $89.17 \%$, ranging $67-100 \%$. His independent task completion during intervention demonstrated a mean change of $74.17 \%$ from baseline, with no overlapping data points. The intervention trend showed an immediate increase from baseline, with an increasing to stable trend across the eight intervention sessions. As demonstrated in Table 3, Kaleb required more error correction in the form of SLP for the flossing task, as he initially struggled with the fine motor aspects of the task. During follow-up, Kaleb had a mean independent task completion of $86.67 \%$, with a complete stable trend. Across the three follow-up sessions, Kaleb chose to complete each of the three sessions without the use of the VP. He correctly performed an average of 13 out of 15 steps without the VP per each follow-up session.

Lastly, Kaleb completed five baseline sessions for the frying an egg task. Kaleb's mean independent task completion during baseline was $8.0 \%$, with a low, stable trend that ranged $7-13 \%$. During intervention Kaleb demonstrated an immediate increase in independent task completion, with a mean change of $83.1 \%$ from baseline, with no overlapping data points. Kaleb's mean independent task completion during intervention was $91.1 \%$, ranging $80-100 \%$, with an increasing to stable trend over the six intervention sessions. During follow-up, Kaleb had a mean independent task completion of $95.56 \%$, ranging $87-100 \%$, 
Fig. 1 Kaleb's percentage of task completion accuracy without the use of SLP

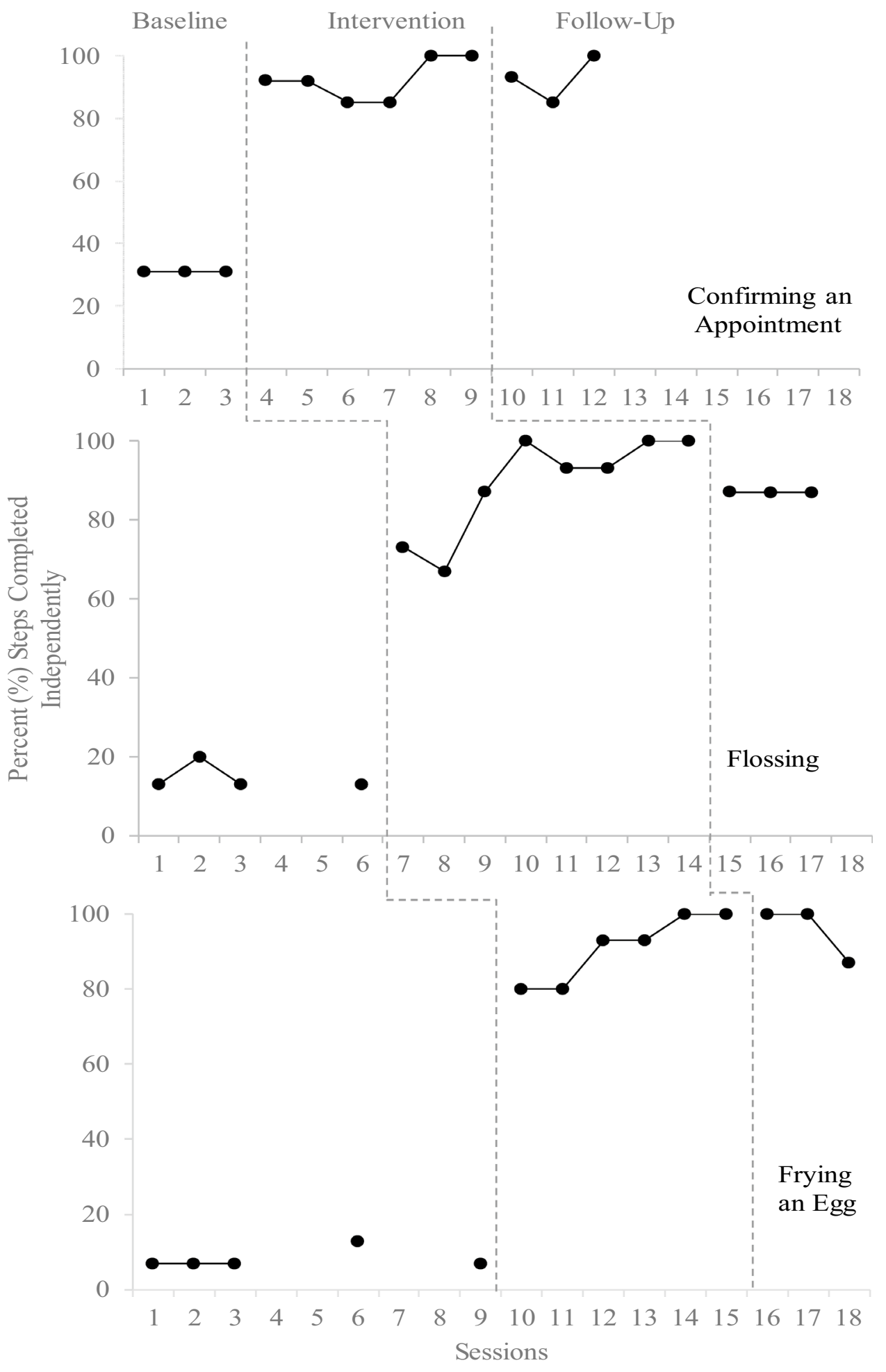

with a stable then decreasing trend. During follow-up, Kaleb chose to complete all sessions without the VP, correctly performing an average of 14.3 out of 15 steps per session $($ range $=13-15)$.

\section{Social Validity}

At the end of the study, the participants met with the authors to discuss what they thought of the study. Kaleb noted that he liked learning new tasks and that VP helped him to get organized so that he could then complete the tasks on his 
Table 3 Percentage of steps requiring SLP and number of sessions to meet mastery criterion per task

\begin{tabular}{lll}
\hline Task & $\begin{array}{l}\text { Mean percentage (\%) of } \\
\text { steps requiring SLP dur- } \\
\text { ing intervention }\end{array}$ & $\begin{array}{l}\text { Number of sessions } \\
\text { required to reach mastery } \\
\text { criterion }\end{array}$ \\
\hline $\begin{array}{l}\text { Confirming } \\
\text { an appoint- } \\
\text { ment }\end{array}$ & $7.7 \%$ & 6 \\
$\begin{array}{l}\text { Flossing } \\
\text { Frying an egg }\end{array}$ & $8.9 \%$ & 8 \\
\hline
\end{tabular}

own after a few attempts with VP. He reported that it was easy to use VP, and that he would be interested in using VP to learn new tasks in the future, depending on the specific task (i.e., a task he was interested in learning). He reported that he did not really like learning the flossing task, in particular, as the fine motor aspect was challenging.

Nora reported that she liked using VP because it was helpful in thinking about how to break down the steps to the task. She noted that this helped her to become more aware of the systematic process of breaking every task into the smallest manageable steps. Nora explained that creating the VP required a lot of work upfront, but that it ultimately freed her from providing live modeling and prompting during the task, which helped her to be more patient, letting her son complete the steps at his own speed. She stated that she was interested in using VP in the future to teach other tasks, especially given the decrease in community-based learning due to the COVID-19 pandemic. More specifically, she

Table 4 Steps correctly completed by the parent to create each VP clip

Steps to create VP clip

Confirming an appoint-

ment

1. Create a task analysis with the research team

$\mathrm{X}$

2. Consider how you want to model the steps, what materials you may need, and what device you want $\mathrm{X}$ to use to record your modeling

3. Gather the materials needed for the task

4. Film your modeling of each step from third-person perspective using the materials and setting that $\quad X$ your child will later use for the task

5. Record an additional 3-5 s blank clip by placing an object in front of the camera so that the screen is $\mathrm{X}$ black (this will later be used as the pause)

6. Download the free app VivaVideo

7. In the VivaVideo app, edit your video by selecting your recording of you modeling the task

8. Use the split function to cut your recording into smaller clips corresponding to each of the discrete, $\mathrm{X}$ task analysis steps

9. Trim or delete any parts of the recording as needed

10. Insert the $3-5 \mathrm{~s}$ blank pause in between each of the steps

11. Mute the original audio

12. Record a voice-over narration of you reading the task analysis step aloud over the corresponding modeling video clip

13. Save the VP to your Photos/Videos folder

Percent accuracy:

$\begin{array}{lll}\mathrm{X} & \mathrm{X} & \mathrm{X} \\ \mathrm{X} & \mathrm{X} & \mathrm{X} \\ \mathrm{X} & \mathrm{X} & \mathrm{X} \\ \mathrm{X} & \mathrm{X} & \mathrm{X} \\ \mathrm{X} & \mathrm{X} & \mathrm{X} \\ \mathrm{X} & \mathrm{X} & \mathrm{X} \\ \mathrm{X} & \mathrm{X} & \mathrm{X} \\ \mathrm{X} & \mathrm{X} & \mathrm{X} \\ & & \\ \mathrm{X} & \mathrm{X} & \mathrm{X} \\ \mathrm{X} & \mathrm{X} & \mathrm{X} \\ \mathrm{X} & \mathrm{X} & \mathrm{X} \\ \mathrm{X} & \mathrm{X} & \mathrm{X} \\ & & \\ \mathrm{X} & \mathrm{X} & \mathrm{X} \\ 100 \% & 100 \% & 100 \% \\ \end{array}$

Table 5 Tau-U effect size trend comparisons for each skill, and the weighted average of all skill trend comparisons

\begin{tabular}{|c|c|c|c|c|c|c|}
\hline \multirow[t]{2}{*}{ Skill } & \multicolumn{3}{|c|}{ Baseline-intervention } & \multicolumn{3}{|c|}{ Baseline-follow-up } \\
\hline & Tau-U & $p$-value & $90 \% \mathrm{CI}$ & Tau-U & $p$-value & $90 \% \mathrm{CI}$ \\
\hline $\begin{array}{l}\text { Confirming an appoint- } \\
\text { ment }\end{array}$ & 1 & 0.0201 & $0.292-1$ & 1 & 0.0495 & $0.162-1$ \\
\hline Flossing & 1 & 0.0066 & $0.395-1$ & 1 & 0.0339 & $0.225-1$ \\
\hline \multirow[t]{2}{*}{ Frying an egg } & 1 & 0.0062 & $0.399-1$ & 1 & 0.0253 & $0.264-1$ \\
\hline & \multicolumn{3}{|c|}{ Tau-U } & \multicolumn{2}{|l|}{$p$-value } & $90 \% \mathrm{CI}$ \\
\hline \multicolumn{2}{|l|}{ Weighted average } & 1 & & 0 & & $0.6306-1$ \\
\hline
\end{tabular}


expressed her interest in teaching Kaleb more independent living and transition skills, such as cooking, cleaning, and grooming skills, to further promote independence and to ensure that he not only completes tasks but does so with a high level of quality. When asked about the drawbacks, Nora reported that the preparation work and learning curve associated with using the video-editing software required a substantial amount of time and energy, but now that she had learned to use it, it may be quicker in the future. In terms of the implementation, she stated that the handouts and guidance from the research team were helpful, but that implementing three different tasks, all in different phases with different procedures and experimental design schedules sometimes got confusing. Nevertheless, she still completed the study procedures with high fidelity, and looked forward to using VP in the future without the constraints of following an experimental design.

\section{Discussion}

The purpose of this study was to examine the effects of a parent-created and parent-implemented VP intervention with SLP strategy on the acquisition and maintenance of daily living skills of an adolescent with ASD. The parent was able to independently create high quality VP clips for three daily living tasks and implement the VP intervention with error correction with high accuracy of fidelity. The adolescent with ASD acquired three target skills following the use of VP with error correction and continued to complete tasks with high levels of accuracy during a follow-up assessment. Social validity interviews indicated that both the parent and adolescent participants valued the use of VP in teaching daily living skills and noted their desire to continue making new VP clips to teach or learn, respectively, further daily living and self-care skills. The findings from this study indicate that parent-created and implemented VP combined with SLP can be an effective strategy to help adolescents with ASD acquire daily living skills in an authentic setting.

This study makes a novel contribution to this line of literature by examining the effects of parent-created and parent-implemented VP, adding to the scarce research on the effects of parent-implemented VP to teach daily living skills to children with ASD (Cruz-Torres et al. 2020) and to the evidence-base on the effects of VP to teach daily living skills to students with ASD without a co-occurring diagnosis of ID (Aljehany and Bennett 2019). It is important to note that parent implementation of VP involved the parent providing directions for the child to watch the VP to complete each task step so that the child operated the VP clip as an independent self-prompting strategy. Although many studies that used VP to teach daily living skills to students with ASD have used instructor-operated (Domire and Wolfe 2015) or parent-operated VP (Cruz-Torres et al. 2020), training students with ASD to use VP as a selfprompting device could add to increased independent functioning when completing daily living tasks. Equipping parents with the skills necessary to create and implement VP can be valuable in helping improve the functional independence of adolescents with ASD as they start to prepare for transition into adult life.

In addition, the parent's use of error correction helped the adolescent child improve the task steps he struggled to complete correctly with VP alone. Although Fig. 1 does not indicate which steps required error correction, the data reflecting Kaleb's accuracy of independent task completion with VP show the immediate effects of VP on his skill acquisition. The mean percentage of steps that required error correction was very small, ranging from 7.7 to $10.8 \%$ across three tasks. Most of the error correction that Kaleb required in the hierarchy of SLP were level one (verbally instructing him to watch the VP clip again) and level two (verbally prompting him on the completion of the task step). On only a few occasions, Kaleb needed modeling and physical prompting due to his challenges with gross motor and fine motor skills. More specifically, he required in-vivo modeling prompt once during the frying an egg task to learn the correct dial placement for "medium low" on the gas stove and required a number of in-vivo modeling prompts during the flossing task to demonstrate how to hold the floss correctly to reach his back teeth. Kaleb only received one physical prompt throughout the intervention phase, again to demonstrate the correct way to hold and manipulate the floss. This may be due to challenges Kaleb experienced with fine motor skills. He willingly accepted his mother's hand-over-hand guidance, and subsequently performed this step better in future sessions.

During follow-up, Kaleb had access to and was told that he could use VP, but he felt that he knew how to complete the tasks and only referred to certain steps of VP for confirming an appointment task when he seemed to hesitate on what to do next. While he chose not to use VP during frying an egg task, he was able to independently fry an edible egg during follow-up sessions. The error analysis showed that during the final follow-up session, he broke the egg yolk while cracking the egg and turned off the stove before egg was fully cooked but let it cook in the residual heat of the pan. While flossing his teeth during follow-up sessions, Kaleb was again able to independently complete the task, but did not get in between each of his teeth in some instances due to challenges with fine motor skills. Overall, the parent's creation and implementation of VP at home to successfully teach her child daily living skills suggest that VP can be an effective strategy for teaching daily living skills in authentic settings. This is especially important when children with ASD start to prepare for transition to post-school life and need to learn daily living skills for adulthood. 
This study also contributes to the literature on parentimplemented interventions, indicating that parents can effectively implement EBPs with high fidelity (Najdowski et al. 2010; Oliver and Brady 2014; Seiverling et al. 2012). The novelty of this study is that the parent learned how to create VP clips and implement VP with error correction to teach daily living skills to her son in a home setting. It is important to note that the focus of our study was not to determine a presence or absence of a causal relation between parent training and parent learning outcomes of creating and implementing VP. Rather, our focus was to determine the presence or absence of a causal relation between parent-created and parent-implemented VP on the child's skill acquisition. Parent training targeted the systematic process for creating VP clips, implementing VP during intervention sessions, and providing error correction with least to most prompting. Since Kaleb was taught how to access and use the VP clips on his computer to complete tasks during the pre-intervention training phase, the parent's implementation of VP consisted of telling Kaleb to watch the VP clips to complete each task and providing error correction, when necessary. Nora created all three VP clips with high quality and implemented the intervention with high fidelity across all tasks (93\% to $100 \%)$. The steps that she missed during intervention implementation were either not providing verbal praise at the end or providing a level of error correction out of the hierarchy of prompting (e.g., providing verbal prompting before telling her son to watch the VP clip again). Furthermore, the procedural reliability steps of the parent's implementation of baseline and follow-up phases (see Table 2) were also high.

Based on systematic reviews and meta-analyses of the literature, the majority of VP studies on teaching daily living skills to students with ASD have been conducted in a school setting with the researcher as the interventionist (e.g., Aljehany and Bennett 2019; Domire and Wolfe 2015; Gardner and Wolfe 2015; Hong et al. 2016). Therefore, the findings from the current study have important implications for research and practice in teaching daily living skills using VP through authentic agents and contexts. While the majority of studies have used instructor-controlled (Domire and Wolfe 2015) or parent-controlled VP (Cruz-Torres et al. 2020), training children to access and operate VP as a self-instructional tool could shift instruction from adult prompting to self-instruction (Shepley et al. 2018). Furthermore, the ease of creating instructional VP clips using everyday technology (Kaleb's parent used her smartphone to create VP clips) and reusing those VP clips in the future when the child may need to refresh his or her memory, can be efficient and effective in helping adolescents with ASD acquire and complete many selfcare and daily living tasks.

\section{Limitations and Directions for Future Research}

There are several limitations which highlight the need for future research. While SCRD can be implemented with one participant and a functional relation can be established by replicating the effects of the intervention across three behaviors, replication of effect across participants (parents and children in this case), settings, and skills enhances the generalizability of findings. Thus, the small number of participants could limit the generalizability of these findings. Further, one child with ASD without a co-occurring diagnosis of ID limits the generalizability of the findings, and replications are needed to enhance the generalizability to children with ASD with cognitive disability. Since we did not explicitly teach Nora how create the task analysis steps, but rather coached her in creating them, her continued use of VP effectively may also depend on creating task analysis steps that are appropriate for the task at hand. Additionally, the parent had a graduate level education (i.e., Master's degree) and was motivated to learn how to create and implement an EBP to support her son's skill acquisition. Therefore, future replication studies with parents of differing education levels and socio-demographic backgrounds are necessary to extend the generalizability of the findings. Future research might examine the implementation of VP at home across various groups of families. Further examining various factors (e.g., family dynamics, parent factors, etc.) that can impact parents' implementation of EBPs in home settings could further add to the literature in this area.

Since the study was conducted in an authentic setting, we examined the maintenance of skills during follow-up only 1 week after the conclusion of the intervention phase. Future studies examining the skill maintenance at longer intervals of time could offer insight into long-term effects of the intervention. Further, we did not examine the generalization across settings as our study took place in an authentic setting. However, it might be of interest for future research to plan for generalization of skills across new contexts and materials when it is developmentally appropriate for a participant who is making plans to live independently at the time of the study. Another limitation is that we did not have Kaleb call different doctor's offices during each session of the study for the confirming an appointment task. It was not practical and realistic to have Kaleb call different doctor's offices when he did not have appointments to confirm. However, it is important to note that while Kaleb followed VP to confirm an appointment according to the steps of the task analysis (see Table 1), the VP modeled the process for some steps that required flexibility from the participant, e.g., during every week of the study, Kaleb had to find the next date and time of the appointment in the future which varied each time, find the number for his doctor's office from the appointment calendar, and say the correct date and time of 
his next appointment. Additionally, Nora implemented the VP per each task once a week to teach daily living skills to Kaleb. While not a limitation of this study, future replications might consider increasing the intensity of the intervention for children with ASD and co-occurring ID.

\section{Conclusion}

The findings of this study support that a parent-created and parent-implemented VP intervention can be effective in teaching daily living skills to adolescents with ASD in home settings. Independent functioning in daily life is an ultimate goal for adolescents with ASD as they grow into adults. VP is one intervention that can be used to further increase independent functioning and shift the instruction from the parent to the adolescent. The time and effort required to develop VP clips for the first time may seem intimidating to parents. Yet, once parents create VP clips and show their child how to self-operate VP to complete daily living tasks, children can use VP as a self-instruction and self-prompting strategy to complete a variety of daily living tasks, furthering increasing their independence as they move toward adulthood.

Author Contributions GY conceived of the study, designed the study, assisted with data collection and analysis, and wrote the paper. BBC served as the primary data collector and assisted with the design, data analysis, and the writing of the paper.

Funding This research did not receive any specific grant from funding agencies in the public, commercial, or not-for-profit sectors.

\section{Compliance with Ethical Standards}

Conflict of interest On behalf of all authors, the corresponding author states that there is no conflict of interest.

Ethical Approval All procedures performed in studies involving human participants were in accordance with the ethical standards of the institutional review board of the University of Maryland, College Park and with the 1964 Helsinki declaration and its later amendments or comparable ethical standards.

Informed Consent Informed consent was obtained from all individual participants included in the study.

\section{References}

Aljehany, M. S., \& Bennett, K. D. (2019). Meta-analysis of video prompting to teach daily living skills to individuals with autism spectrum disorder. Journal of Special Education Technology, 34(1), 17-26. https://doi.org/10.1177/0162643418780495.

Barton, E. E., Lloyd, B. P., Spriggs, A. D., \& Gast, D. L., (2018). Visual analysis of graphic data. In J. R. Ledford, \& D. L. Gast (Eds.), Single case research methodology: Applications in special education and behavioral sciences (3rd ed.). New York: Routledge/Taylor \& Francis Group.

Cruz-Torres, E., Duffy, M. L., Brady, M. P., Bennett, K. D., \& Goldstein, P. (2020). Promoting daily living skills for adolescents with autism spectrum disorder via parent delivery of video prompting. Journal of Autism and Developmental Disorders, 50, 212-223. https://doi.org/10.1007/s10803-019-04215-6.

Domire, S. C., \& Wolfe, P. (2015). Effects of video prompting techniques on teaching daily living skills to children with autism spectrum disorders: A review. Research and Practice for Persons with Severe Disabilities, 39, 211-226. https://doi. org/10.1177/1540796914555578.

Finke, E. H., Davis, J. M., Benedict, M., Goga, L., Kelly, J., Palumbo, L., et al. (2017). Effects of a least-to-most prompting procedure on multisymbol message production in children with autism spectrum disorder who use augmentative and alternative communication. American Journal of Speech-Language Pathology, 26, 81-98. https://doi.org/10.1044/2016_AJSLP-14-0187.

Fleury, V. P., Hedges, S., Hume, K., Browder, D. M., Thompson, J. L., Fallin, K., et al. (2014). Addressing the academic needs of adolescents with autism spectrum disorder in secondary education. Remedial and Special Education, 35, 68-79. https://doi. org/10.1177/0741932513518823.

Gardner, S. J., \& Wolfe, P. S. (2015). Teaching students with developmental disabilities daily living skills using point-of-view modeling plus video prompting with error correction. Focus on Autism and Other Developmental Disabilities, 30, 195-207. https://doi.org/10.1177/1088357614547810.

Gast, D. L., Lloyd, B. P., \& Ledford, J. R. (2018). Multiple baseline and multiple probe designs. In J. R. Ledford \& D. L. Gast (Eds.), Single case research methodology: Applications in special education and behavioral sciences (3rd ed.). New York: Routledge/Taylor \& Francis Group.

Heiman, T. (2002). Parents of children with disabilities: Resilience, coping, and future expectations. Journal of Developmental and Physical Disabilities, 14(2), 159-171. https://doi. org/10.1023/A:1015219514621.

Hong, E. R., Ganz, J. B., Mason, R., Morin, K., Davis, J. L., Ninci, J., et al. (2016). The effects of video modeling in teaching functional living skills to persons with ASD: A meta-analysis of single-case studies. Research in Developmental Disabilities, 57, 158-169. https://doi.org/10.1016/j.ridd.2016.07.001.

Howlin, P., \& Moss, P. (2012). Adults with autism spectrum disorders. The Canadian Journal of Psychiatry, 57(5), 275-283. https://doi.org/10.1177/070674371205700502.

Hughes, E. M., \& Yakubova, G. (2016). Developing hand-held video intervention for students with autism spectrum disorder. Intervention in School and Clinic, 52, 115-121. https://doi. org/10.1177/1053451216636059.

Hume, K., Loftin, R., \& Lantz, J. (2009). Increasing independence in autism spectrum disorders: A review of three focused interventions. Journal of Autism and Developmental Disorders, 39, 1329-1338. https://doi.org/10.1007/s10803-009-0751-2.

Johnson, J. W., Blood, E., Freeman, A., \& Simmons, K. (2013). Evaluating the effectiveness of teacher-implemented video prompting on an iPod touch to teach food-preparation skills to high school students with autism spectrum disorders. Focus on Autism and Other Developmental Disabilities, 28(3), 147-158.

Ledford, J. R., Lane, J. D., \& Gast, D. L. (2018). Dependent variables, measurement, and reliability. In J. R. Ledford \& D. L. Gast (Eds.), Single case research methodology: Applications in special education and behavioral sciences (3rd ed.). New York: Routledge/Taylor \& Francis Group.

Libby, M. E., Weiss, J. S., Bancroft, S., \& Ahearn, W. H. (2008). A comparison of most-to-least and least-to-most prompting on the 
acquisition of solitary play skills. Behavior Analysis in Practice, 1, 37-43. https://doi.org/10.1007/bf03391719.

Najdowski, A. C., Wallace, M. D., Reagon, K., Penrod, B., Higbee, T. S., \& Tarbox, J. (2010). Utilizing a home-based parent training approach in the treatment of food selectivity. Behavioral Interventions, 41, 90-107. https://doi.org/10.1002/bin.298.

Oliver, P., \& Brady, M. P. (2014). Effects of covert audio coaching on parents' interactions with young children with autism. Behavior Analysis in Practice, 7, 112-116. https://doi.org/10.1007/s4061 7-014-0015-2.

Park, J., Bouck, E., \& Duenas, A. (2019). The effect of video modeling and video prompting interventions on individuals with intellectual disability: A systematic literature review. Journal of Special Education Technology, 34, 3-16. https://doi.org/10.1177/01626 43418780464.

Parker, R. I., \& Vannest, K. (2009). An improved effect size for single-case research: nonoverlap of all pairs. Behavior Therapy, 40, 357-367. https://doi.org/10.1016/j.beth.2008.10.006.

Parker, R. I., Vannest, K. J., Davis, J. L., \& Sauber, S. B. (2011). Combining nonoverlap and trend for single-case research: TauU. Behavior Therapy, 42, 284-299. https://doi.org/10.1016/j. beth.2010.08.006.

QuVideo Inc. (2020). VivaVideo (Version 8). https://www.vivavideo. tv/.

Seaman-Tullis, R. L., Cannella-Malone, H. I., \& Brock, M. E. (2019). Training a paraprofessional to implement video prompting with error correction to teach a vocational skill. Focus on Autism and Other Developmental Disabilities, 34(2), 107-117. https://doi. org/10.1177/1088357618794914.

Seiverling, L., Williams, K., Sturmey, P., \& Hart, S. (2012). Effects of behavioral skills training on parental treatment of children's food selectivity. Journal of Applied Behavior Analysis, 45, 197-203. https://doi.org/10.1901/jaba.2012.45-197.

Shepley, S. B. (2017). Self-instructing with mobile technology: Considerations and applications to increase independence.
TEACHING Exceptional Children, 50(2), 59-65. https://doi. org/10.1177/0040059917704971.

Shepley, S. B., Ayres, K. M., Cagliani, R., \& Whiteside, E. (2018). Effects of self-mediated video modeling compared to video selfprompting for adolescents with intellectual disability. Education and Training in Autism and Developmental Disabilities, 53(3), 264-275.

Steinbrenner, J. R., Hume, K., Odom, S. L., Morin, K. L., Nowell, S. W., Tomaszewski, B., et al. (2020). Evidence-based practices for children, youth, and young adults with Autism. The University of North Carolina at Chapel Hill, Frank Porter Graham Child Development Institute, National Clearinghouse on Autism Evidence and Practice Review Team.

Vannest, K. J., Parker, R. I., Gonen, O., \& Adiguzel, T. (2016). Single Case Research: Web based calculators for SCR analysis. (Version 2.0) [Web-based application]. College Station, TX: Texas A\&M University. Retrieved September 21, 2020, from singlecaseresearch.org.

Yakubova, G., Leibowitz, L., Baer, B. L., Halawani, N., \& Lestremau, L. (2019). Self-directed video prompting and least-to-most prompting: Examining ways of increasing vocational skill acquisition among students with autism spectrum disorder and intellectual disability. Advances in Neurodevelopmental Disorders, 3(3), 246-258. https://doi.org/10.1007/s41252-019-0097-5.

Yanardag, M., Birkan, B., Yilmaz, I., Konukman, F., Agbuga, B., \& Lieberman, L. (2011). The effects of least to most prompting procedure on teaching basic tennis skills for children with autism. Kinesiology, 43, 44-55.

Publisher's Note Springer Nature remains neutral with regard to jurisdictional claims in published maps and institutional affiliations. 